\title{
TRANSVERSAL POLARIZATION VECTOR IN LEED FROM Au (110)
}

\author{
M. ERBUDAK, G. RAVANO \\ Laboratorium für Festkörperphysik, ETHZ, CH-8093 Zürich, Switzerland \\ and \\ N. MÜLLER \\ Fachbereich Physik, Universität Osnabrück, D-4500 Osnabrück, Fed. Rep. Germany
}

Received 23 February 1982

We present the first measurements of both transversal components of the spin polarization vector of electrons diffracted from a $\mathrm{Au}$ (110) surface, obtained with an absorption detector. The results clearly demonstrate multiple scattering effects.

The occurrence of spin polarization in low energy electron diffraction (LEED) has been attracting a growing interest. The experimental results, however, are mostly confined to the analysis of the polarization component $P_{n}$ normal to the scattering plane [1]; in electron-atom scattering, this is the only non-trivial component [2]. The existence of the "in-plane" components, both the longitudinal $\left(P_{k}\right)$ and the transversal $\left(P_{e}\right)$ which occur as a consequence of multiple scattering, has been predicted theoretically [3]. Recently, the longitudinal component $P_{k}$ of the polarization vector was measured in LEED at Pt (111), which shows excellent agreement with the calculations [4]. Here, we report on the first measurements of the transversal in-plane polarization component $P_{e}$ of electrons diffracted at a $(2 \times 1)$ reconstructed $\mathrm{Au}$ (110) surface, along with the normal component $P_{n}$ and the diffracted intensity, as a function of the azimuth angle $\phi$, at a constant scattering energy and angle. Thereby the scattering plane contains the surface normal. In agreement with the symmetry considerations in the reflection of an axial vector [5], the rotation diagrams thus obtained show that $P_{e}$ vanishes if a mirror symmetry plane of the crystal coincides with the scattering plane. Then, like in the case of electron-atom scattering, the polarization vector is normal to the scattering plane. The existence of $P_{e}$ or $P_{k}$ is clear evidence for multiple scattering proces- ses away from and not symmetrical to the scattering plane.

The experimental apparatus has been described in detail elsewhere [6]. The essential components used in this work are shown schematically in fig. 1. Unpolarized electrons are incident on the surface of a Au (110) single crystal under an angle of incidence of $\alpha=20^{\circ}$. The specularly reflected beam (OO LEED reflex) is directed into the electrostatic spherical energy analyzer which accepts a solid angle of $6 \times 10^{-4}$ sr and operates at $\Delta E / E=1 \%$. At the exit, the major axes characteristic for the scattering event, $n, k$, and $e$, remain unchanged. They are defined as $n=\left(k_{0}\right.$

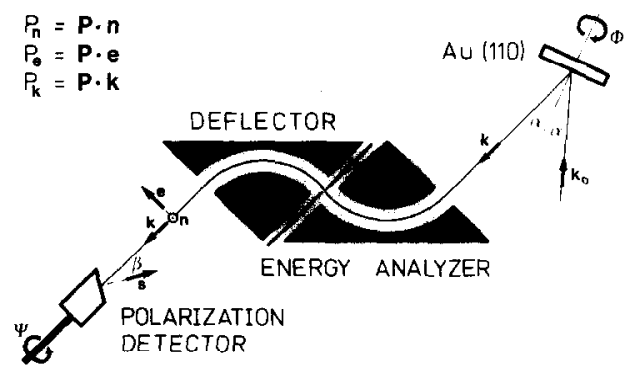

Fig. 1. A schematic of the experiment showing the target of Au single crystal, the spherical energy analyzer, and the absorption detector for electron spin polarization. The plane of electron incidence for the absorber which is shown for $\psi=0^{\circ}$ and the scattering plane coincide with the plane of the figure. 
$\times k) /\left|k_{0} \times k\right|$ and $e=(k \times n) /|k \times n|$, where $k_{0}$ and $k$ are the wave vectors of the incident and diffracted electrons, respectively. Accordingly, $P_{e}=P \cdot e$, $\boldsymbol{P}_{\boldsymbol{n}}=\boldsymbol{P} \cdot \boldsymbol{n}$, and $\boldsymbol{P}_{\boldsymbol{k}}=\boldsymbol{P} \cdot \boldsymbol{k}$. After a beam forming stage (not shown), the electrons hit the polarization detector under an angle of incidence $\beta=30^{\circ}$. The principle of operation of the detector is based on spin dependent electron absorption at gold $[6,7]$; the details have already been communicated [8]. The rotation axis of the absorber (variation of $\psi$ ) coincides with $k$ to keep $\beta$ constant. The components of the polarization, $P_{n}$ and $P_{e}$, are obtained by measuring the absorption current $I(\psi)$ every $90^{\circ}$, with $P_{k}$ being unaccessible. For $\psi=0^{\circ}$ and $\psi=180^{\circ}$, the plane of electron incidence, $k \times s$, where $s$ is the surface normal of the $a b$ sorber, coincides with the scattering plane, $\boldsymbol{k}_{0} \times \boldsymbol{k}$; then the measurement yields the normal component $P_{n}$ of the polarization vector. For a beam of electrons fully polarized along $n$, i.e., $P_{n}=1$, one obtains $1=\left[I\left(180^{\circ}\right)-I\left(0^{\circ}\right)\right] / n I_{0}$, where $I_{0}$ is the intensity of electrons impinging onto the absorber and $\eta$ the sensitivity of polarization detection. Then for any unknown polarization $P_{n}=\left[I\left(180^{\circ}\right)-I\left(0^{\circ}\right)\right] / \eta I_{0}$ and $P_{e}$ $=\left[I\left(270^{\circ}\right)-I\left(90^{\circ}\right)\right] / \eta I_{0}$. The energy of the electrons hitting the absorber is adjusted after their energy analysis such that $I\left(180^{\circ}\right)=-I\left(0^{\circ}\right)$ and equivalently $I\left(270^{\circ}\right)=-I\left(90^{\circ}\right)$. Then, for a beam of unpolarized electrons $I(\psi)=0$. The statistical uncertainty $\Delta P$ in polarization detection is simply $\Delta P=\left(2 / \eta^{2} I_{0}\right)^{1 / 2}$ $[8,9]$.

Fig. 2 presents $P_{n}$ results, as a function of electron

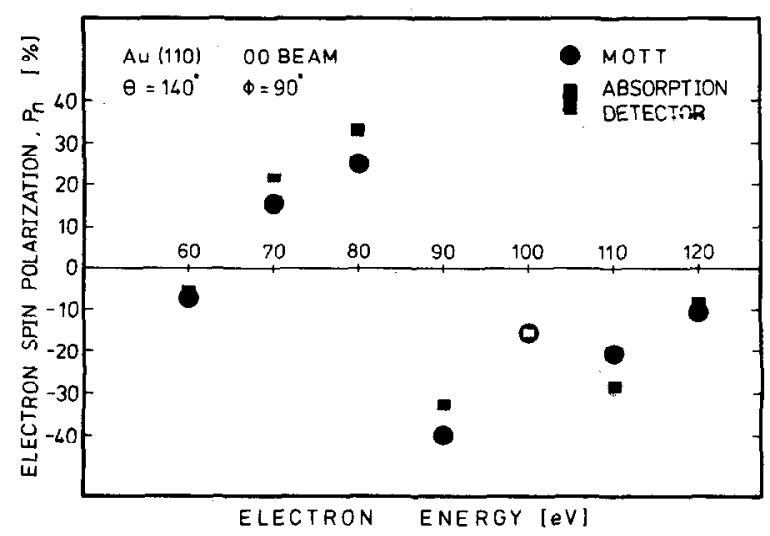

Fig. 2. Electron spin polarization of the OO LEED beam from $\mathrm{Au}(110)$ as a function of the electron energy, measured independently with the absorption detector and with a Mott detector [10]. energy, obtained for the specularly reflected LEED beam from $\mathrm{Au}$ (110). The close packed chains of the surface mesh are perpendicular to the scattering plane $\left(\phi=90^{\circ}\right)$ and the scattering angle is $\theta=140^{\circ}\left(\alpha=20^{\circ}\right)$. Fig. 2 shows polarization values obtained with the absorption detector and compares them with those obtained with a conventional Mott scatterer [10]. The horizontal dimensions of the prints represent the uncertainties in the scattering energy and the vertical ones stand for the errors in the determination of the polarization. The latter are due to the limitations of the present current detection system and further introduced by the rotation of $\psi$; the statistical uncertainty $\Delta P$ is, on the other hand, negligibly small. The overall agreement of these two independent measurements indicates the reliability of the simple absorption detector and yields for its sensitivity $\eta=1.3 \%$ for the gold surface used here. The small deviation in the values at some energies, however, is probably due to the sensitivity of $\boldsymbol{P}_{\boldsymbol{n}}$ on the scattering and azimuth angles, which might be slightly different in the two independent experiments.

Fig. 3 illustrates results of measurements for both components of the polarization vector, $P_{n}$ and $P_{e}$, for $E=100 \mathrm{eV}$ obtained from $\mathrm{Au}(110)$, as a function of the azimuth alignment $\phi, \phi$ is the angle between the mirror symmetry planes of the fcc bulk and the trace of the scattering plane in the surface. The scattering angle is $\theta=140^{\circ}$. Also shown (top) is the intensity distribution of the $\mathrm{OO}$ reflex. The intensity is given in terms of the primary current incident on the gold crystal, without corrections for the transmission function of the energy analyzer. The horizontal dimensions of the data points are due to uncertainties in the azimuth setting $\left( \pm 2^{\circ}\right)$ and the vertical ones present the statistical and instrumental errors in polarization measurements. Solid lines are drawn through the experimental points as an eye guide. Such rotation diagrams are generally preferred to $P(\theta)$ or $P(E)$ profiles because $P(\phi)$ displays the crystal symmetry [11]. For a primary electron beam with the wave vector $k_{0}$ there are many multiple scattering events which lead to $k$. Each partial scattering process occurs with a finite probability and with its own spin state. The diffracted electrons then are a coherent superposition of these partial multiple scattering processes resulting in a spin polarization vector observable in the experiment. The component $\boldsymbol{P}_{\boldsymbol{n}}$ normal to the scattering plane is invariant under reflection of the scattering 


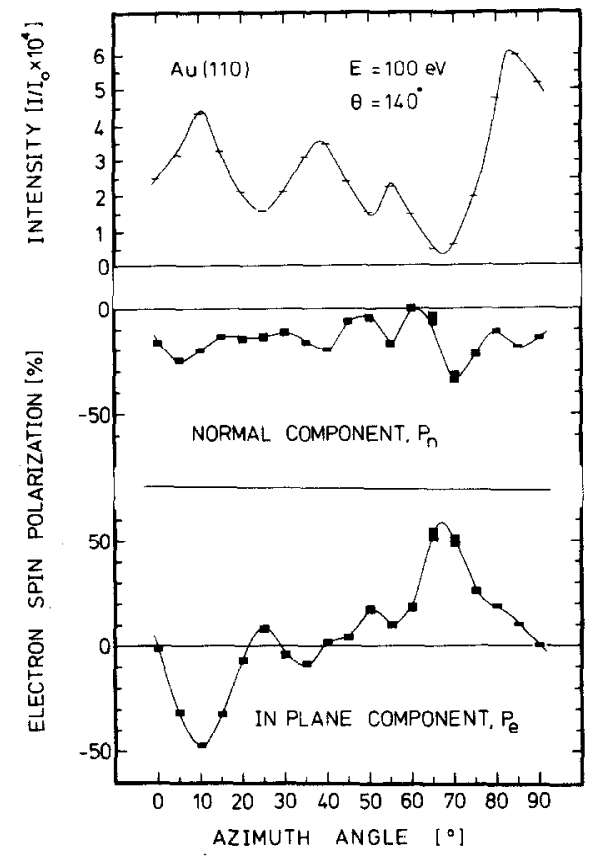

Fig. 3. The intensity $I$, the normal component $P_{n}$, and the inplane component $P_{e}$ for the OO LEED beam from Au (110) as a function of the azimuth angle $\phi$. The scattering angle $(\theta$ $\left.=140^{\circ} \pm 1^{\circ}\right)$ as well as the scattering energy $(E=100 \pm 1 \mathrm{eV})$ are constant. The error bars give the uncertainty in the measurement of polarization and in the determination of $\phi$. Unfortunately, the azimuth interval is limited by the crystal manipulator.

event at a symmetry plane of the crystal where the components in the scattering plane change sign under reflection $[4,5]$. Therefore, the components of the polarization vector in the scattering plane, $P_{e}$ and $P_{k}$, must be zero if the scattering plane containing the surface normal coincides with a mirror plane of the crystal. This is a direct consequence of the conservation of parity under reflection. Only for these special cases, the normal component $P_{n}$ carries the full information about the electron spin polarization. Whenever $P_{e}$ or $P_{k}$ occurs, then partial multiple scattering events asymmetric to the scattering plane play an important role. Throughout the spectrum, $P_{n}$ displays negative values, whereas $P_{e}$ shows an interesting behavior. It is zero for $\phi=0^{\circ}$ and $\phi=90^{\circ}$. In the first orientation the $(00 \overline{1})$ plane of the crystal, containing the close packed chains, coincides with the scattering plane, and in the second case, it is the (110) mirror plane of the bulk. For other azimuth orientations of the crystal, $P_{e}$ has positive and negative values. It even assumes much larger values than the normal component $P_{n}$, as seen in fig. 3. It is interesting that at $\phi=10^{\circ}$ a maximum of the LEED intensity coincides with a maximum of $P_{e}$. For electron-atom scattering maxima in $P$ appear near the intensity $\mathrm{min}$ ima [2]. Such a diffraction condition $[E=100 \mathrm{eV}$, $\theta=140^{\circ}$, and $\phi=10^{\circ}$ for the $O O$ beam from $A u$ (110)] is well suited for a polarization detector based on LEED [1], where $I / I_{0}$ is larger than $4.4 \times 10^{-4}$ and $|A|=|P|=\left(P_{n}^{2}+P_{e}^{2}\right)^{1 / 2}=0.51$.

We have presented the first measurements of the in-plane component of the transversal electron spin polarization vector produced in LEED at $\mathrm{Au}$ (110), measured with a new absorption detector. The results are in accordance with the symmetry properties of the fcc lattice of $\mathrm{Au}$ and show interesting multiple scattering properties asymmetrical to and away from the scattering plane.

The detector for electron spin polarization used in this work, is simpler and more compact than the conventional detection systems [2]. It operates at low electron energies and is compatible with ultra-highvacuum conditions pertinent to surface spectroscopies. Its marked advantage is the easy access to both components of the spin polarization vector, as demonstrated in the present work.

The authors are thankful to H.C. Siegmann for his interest in this work and the Schweizerische Nationalfonds for financial support.

[1] D.T. Pierce and R.J. Celotta, Adv. Electron. Electron Phys. 56 (1981) 219.

[2] J. Kessler, Polarized electrons (Springer, Berlin, 1976).

[3] R. Feder, Surf. Sci. 63 (1977) 283.

[4] P. Baucr, R. Feder and N. Müller, Solid State Commun. 36 (1980) 249; P. Bauer, Report 9/34, MPI für Plasmaphysik, Garching (1980); Thesis, Technische Universität München (1980).

[5] R. Feder, J. Phys. C14 (1981) 2049.

[6] M. Erbudak and G. Ravano, J. Appl. Phys. 52 (1981) 5032.

[7] M. Erbudak and N. Müller, Appl. Phys. Lett. 38 (1981) 575.

[8] M. Erbudak and G. Ravano, 5th General Conf. EPS, Istanbul (1981) Europhys. Conf. Abst. 74 (1981).

[9] D.T. Pierce, S.M. Girvin, J. Unguris and R.J. Celotta, Rev. Sci. Inst. 52 (1981) 1437.

[10] N. Müller, Report 9/23, MPI für Plasmaphysik, Garching (1979); Thesis, Universität München (1977); N. Müller, D. Wolf and R. Feder, Int. Phys. Conf. Ser. 41 (1978) 281.

[11] R. Feder and J. Kirschner, Phys. Stat. Sol. (a) 45 (1978) K 117. 